Article

\title{
Long-Term Evaluation of Autogenous Demineralized Dentin Matrix: A Retrospective 7-Year Clinical Study
}

\author{
Yonsoo Shin. D.D.S., MSD., Young-Kyun Kim. D.D.S., PhD., In-Woong Um. D.D.S. and PhD. \\ 1Department of Oral and Maxillofacial Surgery, Section of Dentistry, \\ Seoul National University Bundang Hospital \\ ${ }^{2}$ Department of Dentistry \& Dental Research Institute, School of Dentistry, Seoul National University, Seoul, \\ Korea \\ ${ }^{3}$ R\&D Institute, Korea Tooth Bank, 622 Eonju-ro, Gangnam-gu, Seoul, Korea \\ * Correspondence:Corresponding author: Young-Kyun Kim, D.D.S., M.S.D., Ph.D. Department of Oral and \\ Maxillofacial Surgery, Section of Dentistry, Seoul National University Bundang Hospital 300, Gumi-dong, \\ Bundang-gu, Seongnam-si, Gyeonggi-do, 463-707, Korea Tel: 82-31-787-7541, Fax:82-31-787-4068. E-mail: \\ kyk0505@snubh.org
}

\begin{abstract}
Autogenous demineralized dentin matrix (ADDM), derived from human extracted tooth, is commonly used as a bone-graft substitute to reconstruct alveolar defects when placing dental implants. The purpose of this retrospective study is to examine efficacy of ADDM in terms of surgical complications and marginal bone resorption by analyzing the medical records and radiographs of patients who received ADDM graft from 2008 to 2011 in our institute. Occurrence of complications, marginal bone loss around implants were investigated with regard to the type of defect, location of bone grafting, and types of bone graft techniques. ADDM-based bone grafting was performed on 221 sites in 82 patients and 208 implants were placed afterwards: The percentage of complications after bone grafting was $15.84 \%$, and the implant survival rate was $95.19 \%$. All complications were resolved with conventional treatment except for the 10 cases of osseointegration failure. The average marginal bone loss was $0.31 \mathrm{~mm}$ at the last examination after the average follow-up period of 7.2 years. Within the limitation of this study, the results of long-term follow-up are consistent with the short-term results of relevant studies. ADDM can produce promising clinical outcomes when used for alveolar ridge augmentation around implants.
\end{abstract}

Keywords: autogenous demineralized dentin matrix; osseointegration; dental implants

\section{Introduction}

The currently used bone graft materials for reconstruction of alveolar bone defects are classified into autogenous, allogenic, xenogenic, and synthetic materials, depending on the origin of the donor bone. The most ideal material for reconstructing hard tissue defects is an autogenous bone graft material that heals quickly and results in osteogenesis, osteoinduction, and osteoconduction, without immune rejection. However, the amount of autogenous bone graft materials that can be obtained by direct extraction is rather limited, and it may cause defects in the donor site. In this respect, Kim at al. developed the autogenous demineralized dentin matrix (ADDM; AutoBT®, Korea Tooth Bank, Seoul, Korea), a bone graft material using an extracted autogenous tooth which produces promising clinical outcomes while showing as good a bone regeneration as from the autogenous bone, for the first time in Korea in 2008. Its clinical safety and efficacy have been proved in Korea, and ADDM is commonly used as bone graft substitute for the defect associated with extraction socket, guided bone regeneration, and sinus augmentation [1-3].

The purpose of the present study is to evaluate the long-term efficacy of ADDM grafts with dental implant retrospectively in terms of the marginal bone resorption around implant, because there has not been a long-term follow up report of ADDM due to its short developmental history= 


\section{Materials and Methods}

The present study was conducted with an approval from the Institutional Review Board of Seoul National University Bundang Hospital (IRB No: B-1910-568-107).

Among the patients who received ADDM graft with dental implant from 2008 to 2011, at the Department of Dentistry, Seoul National University Bundang Hospital, 83 patients (51 men and 32 women; the average age of 51.8) were enrolled in this study. The selected patients for review should meet the requirements as following: 1) Patients whose teeth were extracted and were used to fabricate ADDM required bone graft for implant placement, 2)ADDM were used as primary bone graft material either solely or along with other bone graft materials, 3) Patients have never received radiation therapy and/or chemotherapy in the oral cavity, 4) Patients have taken radiographs such as periapical, panoramic x-ray, or CBCT before and after treatment, 5) All operations were conducted by one expert surgeon. Patients with uncontrolled systemic diseases were excluded. Additional bone graft materials included autogenous, xenogenic, synthetic bones. Bone graft was done in total 221 sites, and 208 implants were placed afterwards. Demographic information of patients, locations of ADDM graft, type of bone graft techniques, and type of graft materials are summarized in Table 1.

Patients who were grafted only using ADDM were designated as the experimental group, while patients who were grafted using ADDM mixed with additional bone graft materials being the control group, defined as ADDM and ADDM + mix group, respectively. Marginal bone resorptions were compared in two groups.

\section{Surgical procedures}

All surgeries were conducted under general anesthesia, intravenous sedative anesthesia or local anesthesia. Incision on the alveolar crest was performed and full flaps were raised afterwards. Bone graft was done by guided bone regeneration, sinus bone graft with lateral or crestal approach, horizontal or vertical ridge augmentation, or ridge split. Implants were placed simultaneously with bone graft or with delayed period to assure sufficient healing, depending on whether primary stability could be obtained or not. In some patients, absorptive barrier membranes or tissue adhesives were used if needed.

\section{Measurements of marginal bone resorption}

We observed the marginal bone loss with panoramic and periapical radiographs from the time of final prosthesis completion as base line to the last follow-up using INFINITT PACS 3.0 (INFINITT Healthcare Co., Ltd. Seoul, Korea) software installed in Orthoceph OC100 CR (Instrumentarium Imaging, Tuusula, Finland), and Heliodent DS (Sirona, Bensheim, Germany) x-ray machine. All periapical radiographs were taken using bisecting angle technique, positioning the tube head in direction of the center of the object. Linear measurement of marginal bone resorption was obtained by one examiner, by calculating the average of mesial and distal distance from implant platform to alveolar crest, which were determined by multiplying the number of exposed threads and pitch distance provided by the manufacturer for each implant, as shown in Figure 1. The amount of bone resorption at the baseline was subtracted from the amount at each time of the measurement to define the baseline as 0 marginal bone resorption. The amount of marginal bone resorption was compared between ADDM and ADDM + mix group. Cases with only sinus bone graft were excluded for the evaluation of the marginal bone resorption since the graft material would not affect crestal margin of the alveolar bone.

\section{Complications}

Complications were classified into surgical complications and complications that occurred after prosthetic loading. The former included wound dehiscence, sinus perforation, and hematoma, while the latter included peri-implant mucositis, peri-implantitis, maxillary sinusitis, and osseointegration failure. Osseointegration failure that occurred within 1 year after bone graft was defined as immediate osseointegration failure, while osseointegration failure that occurred after prolonged period due to any reason such as peri-implantitis was defined as delayed osseointegration failure. 
Prosthetic complications such as cover screw exposure, cover screw fracture, and implant crown fracture were excluded, and thereby only biological complications were covered regarding the relevant issue.

\section{Implant survival and failure}

We defined the state of implant that function without mobility inside oral cavity as implant survival. Any implants which were removed or remained sleeping due to any reason such as osseointegration failure and peri-implantitis were defined as implant failure. Implant survival rate was obtained by above criteria.

\section{Statistical analysis.}

Independent $t$ tests were used to test statistical difference between mean marginal bone resorption of experimental and control groups from the time of final prosthesis completion to the last follow-up. All analyses were performed using software SPSS for Windows (version 17.0, Chicago, IL, USA), and differences were considered to be statistically significant for $\mathrm{P}<.05$.

Table 1. Summary of study variables.

\begin{tabular}{ll}
\hline Study Variable & Descriptive statistics \\
\hline Patients & \\
Sample size (n) & 83 \\
Sex: male & $51(61.45 \%)$ \\
Age & $51.8 \pm 12.20$ \\
Bone grafting sites & \\
Sample size (n) & 221 \\
Locations & $155(70.14 \%)$ \\
$\quad$ Maxilla & $66(29.86 \%)$ \\
$\quad$ Mandible & \\
Types of bone graft techniques & $37(16.74 \%)$ \\
$\quad$ GBR & $52(23.53 \%)$ \\
Sinus bone graft & $30(13.57 \%)$ \\
Ridge augmentation & $2(0.90 \%)$ \\
Ridge split & $100(45.25 \%)$ \\
Combinations & \\
Types of used bone graft materials & $135(61.09 \%)$ \\
ADDM & $86(38.91 \%)$ \\
ADDM + mix &
\end{tabular}

GBR: guided bone regeneration, Sinus: maxillary sinus bone graft by using lateral and crestal approach, Ridge augmentation: horizontal and vertical ridge augmentation, Combinations: combinations of 2 or more bone graft techniques from above. ADDM: bone graft cases using only ADDM, ADDM + mix: bone graft cases using ADDM along with autogenic, allogenic, xenogenic, synthetic bone graft material, or combinations of the above.

\section{Results}

Implants were placed in 73 out of 82 patients after bone grafting, and 208 out of 221 bone graft cases had implants placed. The average follow-up period after bone grafting was 7.2 years, while the average follow-up period after implant prosthesis placement was 6.6 years.

The bone graft technique with guided bone regeneration was performed in 37 cases, the maxillary sinus bone graft technique either with crestal approach or lateral approach in 52, vertical, horizontal, or combined ridge augmentation in 30 , ridge split in 2 , and combinations of the above in 100 cases.

Among the types of bone graft materials, ADDM was used solely in 135 cases and along with other bone graft materials in 86 cases; autogenous bone was used in 11, allogenic bone in 29, 
xenogenic bone in 15 , synthetic bone in 2 , a combination of the autogenous bone and the allogenic bone in 8 , a combination of the autogenous bone and the xenogenic bone in 4 , a combination of the autogenous bone and the synthetic bone in 3 , a combination of the allogenic bone and the xenogenic bone in 13 , and a combination of the allogenic bone and the synthetic bone in 1 case.

Surgical complications after bone grafting included 9 and 3 wound dehiscence, 7 and 14 maxillary sinus perforations in experimental and control group, respectively, and 2 hematomas occurred only in the control group. Complications that occurred after prosthetic loading included 1 and 3 peri-implant mucositis, 13 and 8 peri-implantitis, 5 and 3 osseointegration failures in the experimental and control group, respectively, and 1 maxillary sinusitis occurred only in the control group. All complications, except the cases where implants were removed or remained sleeping due to immediate or delayed osseointegration failure, were resolved with treatments such as peri-implant curettage, medication, and prosthetic re-fabrication.(Table 2)

The average marginal bone loss of $0.09 \mathrm{~mm}, 0.15 \mathrm{~mm}, 0.22 \mathrm{~mm}$, and $0.30 \mathrm{~mm}, 0.07 \mathrm{~mm}, 0.13 \mathrm{~mm}$, $0.25 \mathrm{~mm}$, and $0.49 \mathrm{~mm}$, and $0.08 \mathrm{~mm}, 0.14 \mathrm{~mm}, 0.23 \mathrm{~mm}$, and $0.31 \mathrm{~mm}, 1$ year after prosthesis functioning, 2 years after prosthesis functioning, 3 years after prosthesis functioning, and at the last follow-up, were observed in the experimental, control, and total group, respectively. The marginal bone resorption was within range of minimum 0 to maximum $3.70 \mathrm{~mm}$ and 0 to $5.15 \mathrm{~mm}$ in the experimental and control group, respectively. There was no significant difference in the average marginal bone loss between two groups at all times of the measurement. Average marginal bone loss and standard deviations compared by group and time are shown in table 3. (Fig. 2) The implants' survival rate was $94.81 \%, 96.51 \%$, and $95.19 \%$ for the control, experimental, and total group, respectively. Implant failure was observed in 10 out of 208 cases and occurred in 5 out of 73 patients.

Table 2. Complications and implant survival rates.

\begin{tabular}{ll}
\hline Study Variable & Descriptive statistics \\
\hline ADDM (n=135) & \\
Surgical complications & $16(11.85 \%)$ \\
Wound dehiscence & 9 \\
Sinus perforation & 7 \\
Complications after prosthetic loading & $19(14.07 \%)$ \\
Peri-implant mucositis & 1 \\
Peri-implantitis & 13 \\
Immediate osseointegration failure & 5 \\
Implant survival & $128(94.81 \%)$ \\
\hline ADDM + mix (n=86) & \\
Surgical complications & $19(22.09 \%)$ \\
Wound dehiscence & 3 \\
Sinus perforation & 14 \\
Hematoma & 2 \\
Complications after prosthetic loading & $15(17.44 \%)$ \\
Peri-implant mucositis & 3 \\
Peri-implantitis & 8 \\
Maxillary sinusitis & 1 \\
Immediate osseointegration failure & 3 \\
Implant survival & $83(96.51 \%)$ \\
\hline
\end{tabular}


Table 3. Average marginal bone loss and standard deviations compared by group and time (mm).

\begin{tabular}{ccccc}
\hline & $\begin{array}{c}\text { ADDM } \\
(\mathbf{n}=\mathbf{1 1 0})\end{array}$ & $\begin{array}{c}\text { ADDM + mix } \\
(\mathbf{n}=\mathbf{4 3})\end{array}$ & $\begin{array}{c}\text { Total } \\
(\mathbf{n}=\mathbf{1 5 3})\end{array}$ & \multirow{2}{*}{ Difference } \\
\cline { 2 - 4 } & Mean \pm SD & Mean \pm SD & Mean \pm SD & \\
Baseline & 0 & 0 & 0 & $\mathrm{P}=.819$ \\
1 year & $0.09 \pm 0.41$ & $0.07 \pm 0.44$ & $0.08 \pm 0.42$ & $\mathrm{P}=.825$ \\
2 year & $0.15 \pm 0.56$ & $0.13 \pm 0.56$ & $0.14 \pm 0.56$ & $\mathrm{P}=.825$ \\
3 year & $0.22 \pm 0.76$ & $0.25 \pm 0.82$ & $0.23 \pm 0.77$ & $\mathrm{P}=.132$ \\
\hline At the last follow-up & $0.30 \pm 0.85$ & $0.49 \pm 1.29$ & $0.31 \pm 0.90$ & \\
\hline
\end{tabular}

$\mathrm{P}$ values, independent $\mathrm{t}$ test for difference between ADDM and ADDM + mix groups.

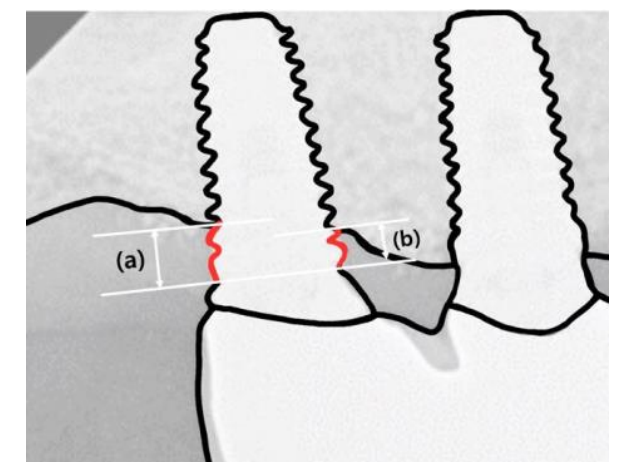

Figure 1. Example of measuring the amount of marginal bone resorption. (a) The number of exposed threads: 2, pitch distance: $0.7 \mathrm{~mm}$, the amount of marginal bone loss: $2 \times 0.7=1.4 \mathrm{~mm}$. (b) The number of exposed threads: 1.5 , pitch distance: $0.7 \mathrm{~mm}$, the amount of marginal bone loss: $1.5 \times 0.7=1.05 \mathrm{~mm}$. Therefore, total marginal bone loss of this example can be obtained as $1.23 \mathrm{~mm}$, the average of distal and mesial marginal bone loss.

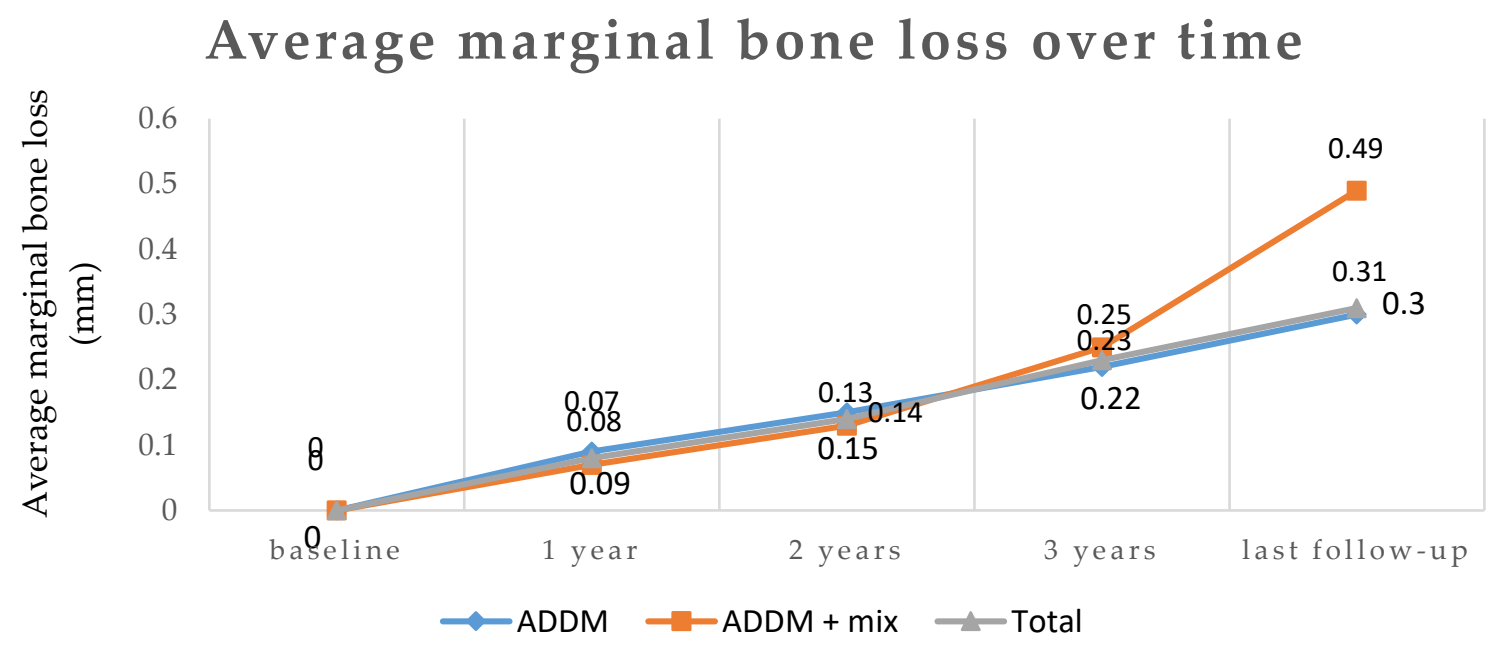

Figure 2. Average marginal bone loss over time. Baseline: marginal bone loss at the time of prosthesis completion, 1 year: marginal bone loss 1 year after prosthesis functioning, 2 years: marginal bone loss 2 years after prosthesis functioning, 3 years: marginal bone loss 3 years after prosthesis functioning, last follow-up: marginal bone loss at the last follow-up, average follow-up period of 6.6 years after prosthesis functioning. No significant difference was found between two groups. 

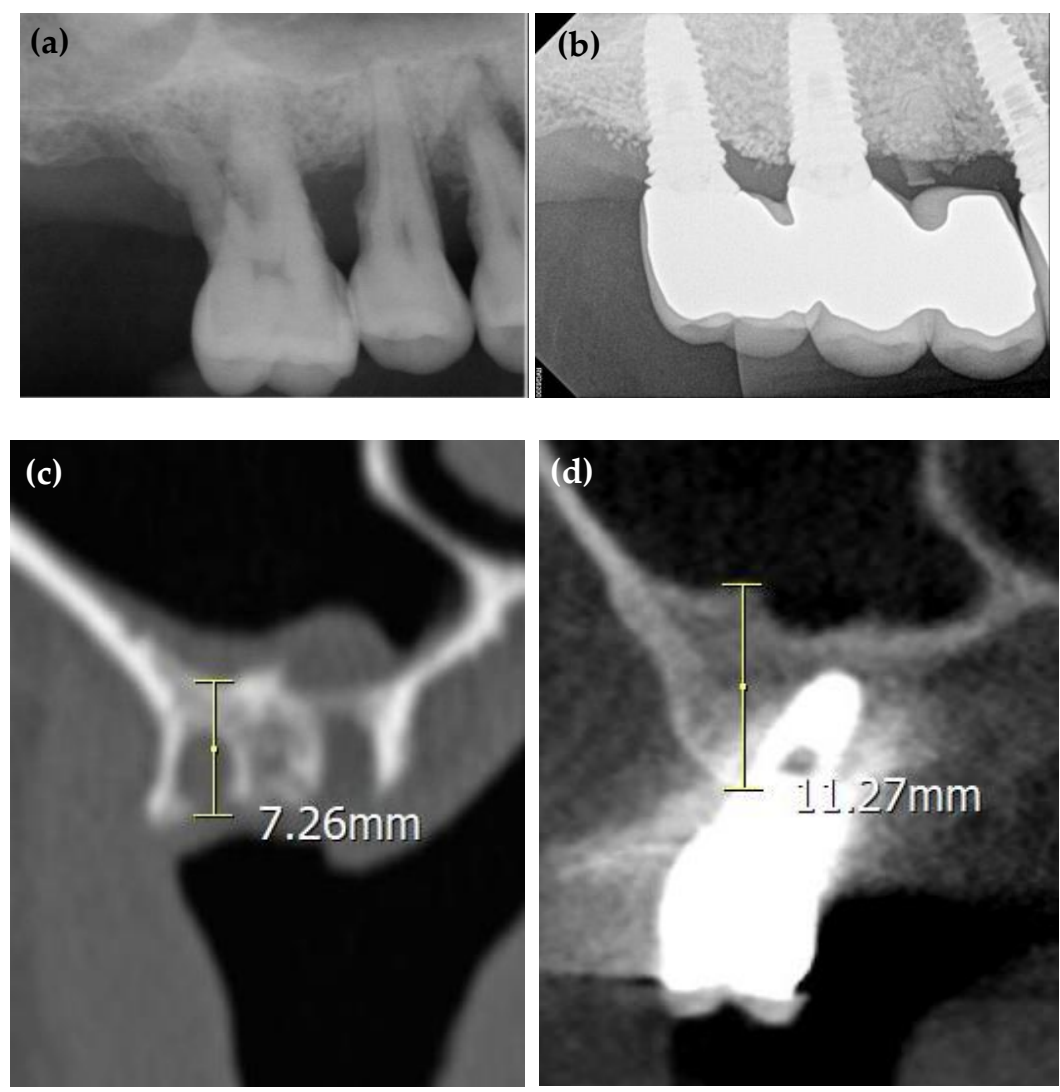

Figure 3. Radiographs of the patient treated with ADDM. (a) Periapical radiograph before extraction. Patient had severe alveolar bone resorption due to periodontitis and maxillary sinus pneumatization. Extracted teeth were processed to produce ADDM. (b) Periapical radiograph after bone graft and implantation. GBR for socket preservation and maxillary sinus bone graft by lateral approach were done with implantation. (c,d) Computed tomography of treated site after extraction and at the last follow-up. Increased radiopacity of the grafted site is observed. The displayed numbers are shown just to demonstrate reference for the rough figure of bone level difference, and are not precise value.

\section{Discussion}

Average 7.2 years of follow-up period had been completed on 208 implants placed after 221 ADDM-based bone grafting. The percentage of complications after bone grafting was $15.84 \%$, and the implant survival rate was $95.19 \%$ (ADDM vs ADDM Mix). All complications were resolved with conventional treatment except for the 10 cases which had implant fixtures removed or remained sleeping due to immediate or delayed osseointegration failure. The average marginal bone loss was $0.59 \mathrm{~mm}$ at the last examination after the average follow-up period of 7.2 years.

The average marginal bone loss of experimental and control groups showed no significant difference after more than 3 years of follow-up, indicating that ADDM can be used with or without other bone graft materials presenting clinical stability. Also, no marginal bone loss at all occurred in 127 cases out of 153 implants which had been followed up for more than 5 years. Implants with ADDM-based bone grafts showed a higher level of marginal bone stability and survival rate. The long-term follow-up findings that the ADDM used for implant treatment kept the alveolar crestal bone stable and showed a higher implant survival rate suggest ADDM's excellent bone regenerative effect, remodeling, and efficacy.(Fig. 3) Cases with only sinus bone graft were excluded for the marginal bone resorption analysis, assuming that ADDM would not affect the marginal bone resorption since the graft site would not involve alveolar crest.

Among a total of 221 bone graft cases, there were various complications, such as maxillary sinus perforation, hematoma, partial wound dehiscence, peri-implantitis, peri-implant mucositis, maxillary sinusitis, and osseointegration failure. Implant failure was observed in 10 out of 208 implants, and occurred in 5 out of 73 patients. 9 failures were due to immediate osseointegration 
failure which occurred within 1 year after implant fixture placement and occurred in all five patients. Another one failure was due to delayed osseointegration failure following severe peri-implantitis, which occurred in one of the five patients. Every implant which undergone osseointegration failure was removed except for one immediate osseointegration failure case which had the fixture remained sleeping under mucosa without functioning.

All 221 bone graft cases showed normal healing without any foreign body rejection. Kim et al. reported in their previous study that the ADDM can be used as a bone graft material regardless of the donor tooth's condition and showed outstanding osteoinduction and osteoconduction as it achieved good bone healing [4]. If the ADDM is not sufficient for a large bone defect, it can be used in combination with other bone graft materials. The present study used allogenic, xenogenic, synthetic, or a combination of these three materials.

Present study used 4 types of bone graft techniques (guided bone regeneration, maxillary sinus bone graft, ridge augmentation, and ridge split). Many published studies also reported that the ADDM was used in various bone graft techniques and produced outstanding outcomes [5-8].

Previous relevant short-term studies about stability of powder and block type ADDM graft showed average marginal bone resorption of $0.20 \mathrm{~mm}$ and $0.70 \mathrm{~mm}$, respectively, while present study showed $0.59 \mathrm{~mm}$ of average marginal bone resorption, results being consistent [9,10]. Compared to several other bone graft materials, ADDM showed similar or superior outcomes regarding marginal bone loss and implant survival rate. Kim et al. reported 0.7-1.0mm of marginal bone resorption after 20-month follow-up of mixed graft of autogenous bone and bovine bone with survival rate of $61-100 \%$ [11]. Ungor et al. reported $1.20 \mathrm{~mm}$ of marginal bone resorption after 30 -month follow-up of allogenic bone graft with survival rate of $100 \%$ [12]. Also, previous relevant studies have shown that ADDM grafting increases bone volume effectively [13]. Grafted sites in this study as well demonstrated increase of bone remodeling as shown by increase of radiopacity in computer tomography.

This study presented few limitations: Only 2- dimensional radiographic data such as panoramic and periapical radiography were used to assess ADDM's marginal bone stability around implants. Since the study was designed to evaluate ADDM's efficacy retrospectively, not all patients had CBCT taken before and after treatment, which made it difficult to assess ADDM's bone regenerative ability and marginal bone stability 3-dimensionally. Further research based on CBCT data is required. Also, this study could not distinguish ADDM's effect from other personal patient factors. Initial clinical effect of ADDM can be distinguished by the quantity and quality of bone graft in the first 4 to 6 months of treatment $[9,13,14]$. Due to its complexity of applying several parameters based on patients retrospective medical records, this study could only adopt marginal bone resorption over other personal factors for long-term results, which is one of the limitations awaiting resolution. To evaluate effects of other factors on ADDM's results, prospective study must be designed and conducted in the future. Another limitation of this research was that the sample size of block and putty type ADDM was relatively small, due to its later development time compared to powder type ADDM. Further study with larger sample size is needed to demonstrate clinical efficacy of each types.

\section{Conclusion}

The long-term follow-ups of ADDM-based bone grafts and suggest the following conclusions:

1. Implants placed after ADDM-based bone grafting showed a high level of marginal bone stability and implant survival rate, and;

2. Complications that occurred in implants placed after ADDM-based bone grafting could be solved by appropriate treatment without causing any serious issues.

3. Long-term results of ADDM according to the donor tooth's condition, type of materials (block, powder, and putty), and type of surgery should be evaluated with more samples and longer follow-up period.

\section{References}


1. Kim, Y.K. Development of autogenous teeth bone graft material and clinical evaluation. J Korean Dent Assoc 2011, 49, 159-169.

2. Kim, Y.K.; Kim, S.G.; Byeon, J.H.; Lee, H.J.; Um, I.U.; Lim, S.C.; Kim, S.Y. Development of a novel bone grafting material using autogenous teeth. Oral Surg Oral Med Oral Pathol Oral Radiol Endod 2010,109, 496503.

3. Um, I.W.; Kim, Y.K.; Mitsugi, M. Demineralized dentin matrix scaffolds for alveolar bone engineering. J Indian Prosthodont Soc 2017, 17, 120-127.

4. Ahn, G.A.; Kim, Y.K.; Um, I.W.; Kim, J.Y. Evaluation of prognosis of autogenous tooth bone graft material according to the condition of donor tooth. J Dent Implant Res 2015, 34, 7-11.

5. Park, S.M.; Um, I.W.; Kim, Y.K.; Kim, K.W. Clinical application of auto-tooth bone graft material. J Korean Assoc Oral Maxillofac Surg 2012, 38, 2-8.

6. Han, M.; Lee, J.K. Clinical study on the efficacy of the autogenous tooth bone graft material (AutoBT). J Korean Assoc Maxillofac Plast Reconstr Surg 2013, 35, 221-226.

7. Um, I.W.; Ku, J.K.; Kim, Y.K. The retrospective clinical study of the autogenous tooth block bone graft. J Dent Implant Res 2015, 34, 27-34.

8. Lee, J.Y.; Kim, Y.K.; Yi, Y.J.; Choi, J.H. Clinical evaluation of ridge augmentation using autogenous tooth bone graft material: Case series study. J Korean Assoc Oral Maxillofac Surg 2013, 39, 156.

9. Kim, Y.K.; Lee, J.H.; Um, I.W.; Cho, Woo-Jin. Guided Bone Regeneration Using Demineralized Dentin Matrix: Long-Term Follow-Up. J Oral Maxillofac Surg 2016, 74, 515.e1-515.e9.

10. Kim, Y.K.; Pang, K.M.; Yun, P.Y.; Leem, D.H.; Um, I.W. Long-term follow-up of autogenous tooth bone graft blocks with dental implants. Clin Case Rep 2017, 5, 108-118.

11. Kim, Y.K.; Yun, P.Y.; Kim, S.G.; Kim B.S., Ong, J.l. Evaluation of sinus bone resorption and marginal bone loss after sinus bone grafting and implant placement. Oral Surg Oral Med Oral Pathol Oral Radiol Endod 2009, 107, 21-28.

12. Ungor, C.; Guven, A.; Songur, T.; Dayisoylu, E.; Kurt, H.; Tosun, E.; Senel, F.C. Marginal bone resorption around dental implants placed in grafted sinuses; an up-to-30-month clinical and radiological follow-up. J Pak Med Assoc 2016, 63, 1124-1128.

13. Pang, K.M.; Um, I.W.; Kim, Y.K.; Woo, J.M.; Kim, S.M.; Lee, J.H. Autogenous demineralized dentin matrix from extracted tooth for the augmentation of alveolar bone defect: a prospective randomized clinical trial in comparison with anorganic bovine bone. Clin. Oral Impl. Res 2017, 28, 809-815.

14. Jung, G.U.; Jeon, T.H.; Kang, M.H.; Um, I.W.; Song, I.S.; Ryu, J.J.; Jun, S.H.; Volumetric, Radiographic, and Histologic Analyses of Demineralized Dentin Matrix Combined with Recombinant Human Bone Morphogenetic Protein-2 for Ridge Preservation: A Prospective Randomized Controlled Trial in Comparison with Xenograft. Materials 2018, 8,1288. 